\title{
ACADÉMIE DE NEUGHATEL
}

\section{LOUIS AGASSIZ}

\section{ET SON SÉJOUR A NEUCHATEL}

DE 1832 A 1846

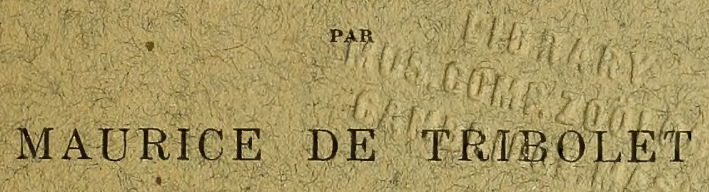

Professeur à la Faculté des Sciences.

NEUCHATEL

IMPRIMERIE ATTINGER FRÈRES

$19 \circ 7$ 
Neve

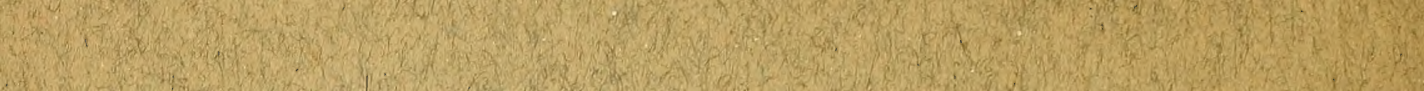

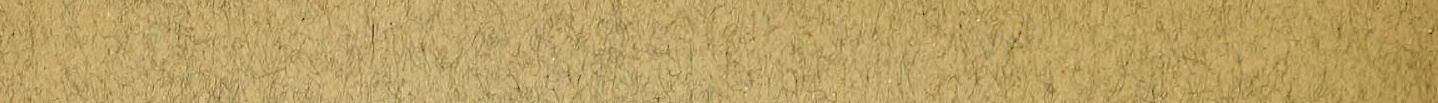
45. b.

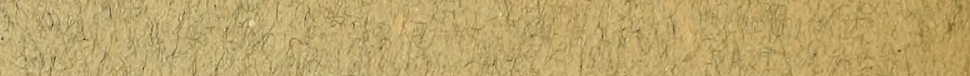

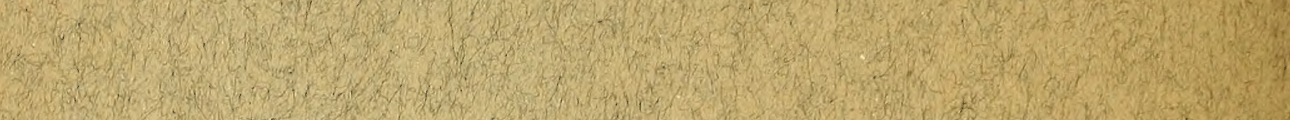
7.

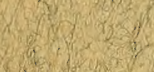

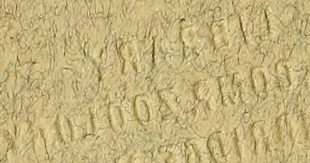
1. (1)

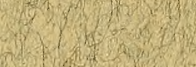

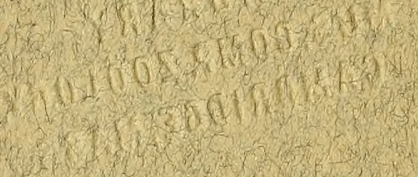

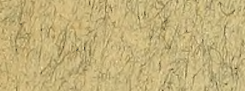
$y^{2} x^{2}$

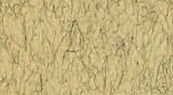

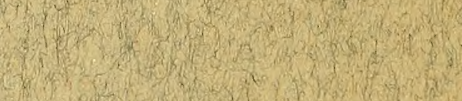

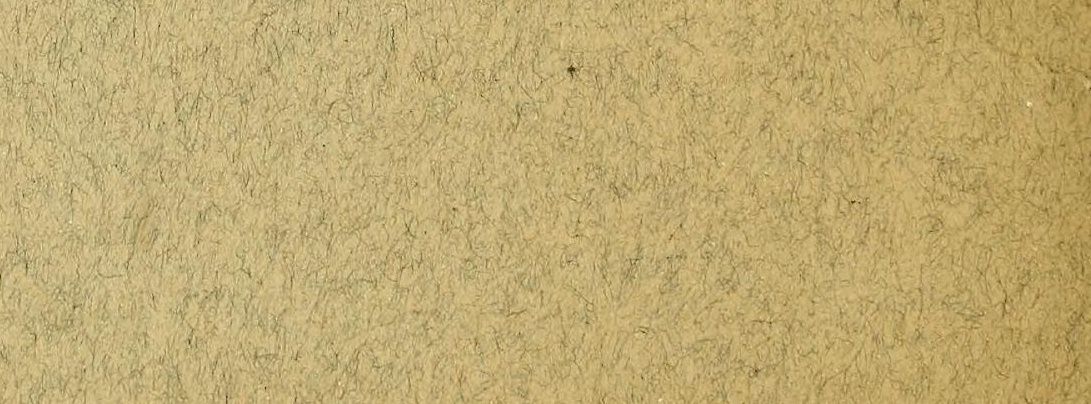

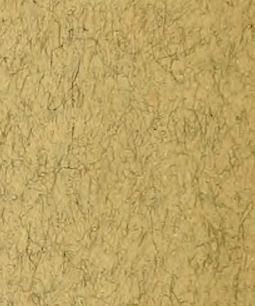

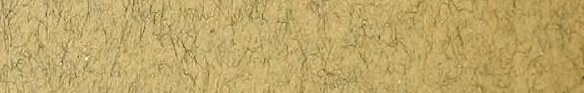
$8 x^{3}$ 


\title{
ACADÉVIE DE NEUCHATEL
}

\section{LOUIS AGASSIZ}

\section{ET SOI SÉJOUR A NEUCHATEL}

\author{
DE 1832 A 1846
}

PAR

\section{MAURICE DE TRIBOLET}

Professeur à la Faculté des Sciences.

NEUCHATEL

IMPRIMERIE ATTINGER FRÈRES

I 907 


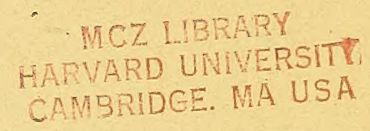




\title{
LOUIS AGASSIZ
}

\section{ET SON SÉJOUR A NEUCHATEL}

\author{
DE .1832 A 18461
}

Il y a aujourd'hui un siècle que naissait non loin d'ici, sur terre fribourgeoise, dans la maison de cure de Môtier en Vully, Jean-Louis-Rodolphe Agassiz. Si le canton de Vaud, auquel il appartient de par sa naissance, dans un élan de pieux enthousiasme, a tenu à célébrer cet anniversaire, Neuchâtel de son côté n'a pas voulu rester en arrière. La Société des Sciences naturelles et l'Académie ont tenu à s'associer elles aussi à cette manifestation, en rendant un hommage public d'admiration et de gratitude à la personne du grand naturaliste qui fut un des fondateurs de la première et une des illustrations de la seconde.

Agassiz, en effet, est un des nôtres. Ill nous appartient par l'œurre qu'il a accomplie chez nous, par les travaux qu'il y a exécuté et par les souvenirs qu'il y a laissé. En dépit de son origine, il est une des plus belles gloires de notre petite pa-

1 Discours prononcé dans la Salle circulaire du Collège latin, le 28 mai r 907 , à l'occasion du centième anniversaire de sa naissance. 
trie dont il se plaisait lui-même à se considérer comme un de ses enfants. Il représente une époque unique de notre histoire, l'éveil de l'enthousiasme pour la science coüncidant avec les débuts de l'enseignement scientifique à Neuchâtel. Aussi était-il bien digne d'avoir sa place dans notre Académie, cet homme qui a aimé par dessus tout la jeunesse et lui a consacré sa vie entière. Grâce, d'un còté, à une décision de nos autorités cantonales, prise au lendemain de sa mort, de l'autre, grâce à la généreuse intervention d'une partie de notre jeunesse académique, son souvenir se trouve chaque jour rappelé à notre mémoire par le pinceau du peintre et le ciseau du sculpteur, qui l'un et l'autre ont fidèlement reproduit ses traits et immortalisé sa figure.

La vie d'Agassiz présente un vif intérèt, mais pour er parler je rencontre une difficulté d'un genre particulier. Que dire, en effet, de nouveau sur sa personne ou sur son ouvre qui n'ait déjà été raconté dans les nombreux articles de journaux et de revues, et dans les multiples biographies qui ont été publiées aussi bien pendant sa vie que surtout après sa mort. Je réclame pour cette lecture votre bienveillante indulgence. J'ai fait de mon mieux et en m'acquittant de la tàche qui m'incombe, je puis dire qu'il m'a été doux, dans un jour anniversaire comme celui-ci, de me sentir posséder le privilège de vous parler de la vie et des œuvres de celui qui fut une des illustrations dont un petit pays comme le nôtre peut à juste titre s'honorer. Heureusement, dans cette admirable vie de travail qu'a été la vie d'Agassiz, il y a assez d'aspects accessibles à tous pour qu'il soit permis, mème à quelqu'un qui ne l'a point connu et ne saurait le juger que du dehors, de célébrer avec sincérité sa mémoire.

La carrière d'Agassiz, si on fait abstraction du temps de son enfance, comprend deux périodes distinctes d'une longueur presque égale, à peu près un quart de siècle chacune, 
la première avant, la seconde après son départ pour le Nouveau Monde.

Pendant la première période, où il se trouvait dans toute la fraicheur de la jeunesse et toute la vigueur de cet âge d'or du talent, il fit dans le domaine encore peu connu de la paléontologie ces investigations qui l'ont placé au premier rang des hommes scientifiques de son temps. C'est aussi dans cette période qu'il commença ses recherches zoologiques, qui n'arrivèrent que plus tard à leur point culminant, et qu'il exposa la conception hardie d'une ère glaciaire universelle, qui aurait été la clòture des temps géologiques, et à laquelle son nom reste perpétuellement attaché.

Dans la seconde période, poursuivant ses travaux avec une ardeur nouvelle sur un théâtre plus vaste, admirablement approprié à sa puissance intellectuelle, cet homme nous apparait comme le grand maître des sciences naturelles, non seulement auprès de ceux qui sont chargés de les enseigner, mais auprès d'une nation toute entière. La sympathie générale et une assistance efficace faisaient encore défaut à ce genre d'études. Dès son arrivée, Agassiz adressa à la nation de chauds appels auxquels elle répondit généreusement. L'école de Cambridge, le Musée Agassiz, l'école d'histoire naturelle d'Anderson, dans l'île de Penikese, comme aussi l'intérêt éveillé universellement en faveur de la science, sont les monuments durables de l'influence bienfaisante qu'il a exercée dans sa seconde patrie.

Au commencement du XVIIIe siècle, quelques hommes, entrainés dans le courant scientifique qui commençait à se faire jour chez nous et qui allait répandant de plus en plus le goût du raisonnement et de l'observation, cultivaient avec amour les premiers germes de nos progrès intellectuels et apportaient leur contingent au progrès général. Ils ensemençaient avec ardeur un sol qui n'était point destiné à rester ingrat, 


\section{$-6-$}

puisqu'il devait plus tard nourrir le génie de savants que nous avons le légitime orgueil de compter comme nos concitoyens.

Au temps de Bourguet ( $1678-1742$ ), notre pays comptait plusieurs savants étroitement liés, formant une petite société dont les investigations s'étendaient sur tout le Jura central, et qui se communiquaient mutuellement leurs observations et leurs découvertes, posant ainsi les premiers jalons des études scientifiques dans notre pays. C'étaient le pasteur Cartier, de la Chaux-du-Milieu ( 7 . . - 759 ), le Dr Abram Gagnebin, de la Ferrière (1707-1800), Élie Bertrand (1713-1797), qui constituent le germe de ce que je pourrais appeler l'école géologique neuchàteloịse, qui devait plus tard rendre à la science les services que l'on connait; c'était le Dr Jean-Antoine d'Ivernois ( $1703-1764)$, sous lequel J.-J. Rousseau, réfugrié au Val-de-Travers, prit le goût de la science qui devait lui sourire et le consoler dans sa vie d'exil.

Ces quelques hommes, mettant en commun, avec simplicité et franchise, leurs observations, leurs découvertes, résumaient en quelque sorte les amis des études au XVIIIe siècle. Exemples d'initiative et d'activité féconde dans le domaine du vrai et du bien, mais voguant au hasard sur la mer de l'inconnu, ils n'en exercèrent pas moins une influence remarquable. Leurs travaux devaient devenir le point de départ d'un mouvement intellectuel dont ils ne pouvaient entrevoir la portée et qui, un demi-siècle plus tard, transformait notre pays.

Louis Bourguet n'était pas Neuchàtelois d'origine. Il était comme les Coulon, comme Ed. Desor, ainsi que tant d'autres familles qui se sont distinguées chez nous par leur intelligence, leur activité, leurs vertus, une victime de la révocation de l'Édit de Nantes. C'était comme Albert de Haller une de ces organisations vraiment encyclopédiques, telles que le XVIII siècle en présente plusieurs exemples. Tour à tour 
littérateur, philosophe, mathématicien, naturaliste, entretenant sans cesse avec les savants de son époque des rapports étroits, il créa autour de lui, comme Louis Agassiz un siècle plus tard, une véritable atmosphère scientifique. Il fut plus et mieux qu'un vulgarisateur, il fut un initiateur. Son ceuvre marque une des étapes de l'histoire scientifique de notre pays et son nom mérite qu'on ne le laisse pas tomber dans l'oubli, mais au contraire qu'on le rappelle quelquefois comme celui de l'homme qui le premier planta d'une main ferme sur notre sol le drapeau de la science, drapeau sur les plis duquel sont venus, dans la suite, s'inscrire bien des noms connus.

Lorsque Bourguet et ses compagnons eurent disparu, l'élan provoqué par eux en faveur des sciences naturelles ne se ralentit point; il devail se perpétuer à Neuchâtel et produire dans la première moitié du siècle suivant la féconde école dont Agassiz fut le chef et la gloire.

A Neuchâtel, comme ailleurs en Suisse, la première moitié du XIXe siècle est marquée par un réveil intellectuel accentué. Le mouvement scientifique créé sous l'influence de Cuvier devait avoir son retentissement chez nous. Avant r 83o, on peut dire que tout était à créer dans ce domaine. Çà et là se montraient bien quelques personnes désireuses de s'intéresser au progrès des sciences et soucieuses d'étudier l'histoire naturelle de leur pays. Mais elles étaient sans lien commun et partant sans un appui qui leur aurait été pourtant si nécessaire. A cette époque, les sciences n'étaient pas enseignées à Neuchâtel, où les études littéraires seules ou presque seules dominaient. On n'avait alors d'autre ambition que celle de préparer, en vue de leurs études universitaires, les jeunes gens qui se destinaient à suivre la carrière d'avocats, de médecins et surtout de pasteurs.

Mais finalement un courant nouveau se manifeste chez nous et les Conseils de la Bourgeoisie se décident à faire quelques 
concessions à l'enseignement scientifique, qui prenait dans le monde une place et une influence prépondérantes. Il en résulta la création de deux chaires, l'une de mathématiques (I 823), l'atitre de physique et de chimie (I83 r). Mais jusque là personne n'avait songé à l'enseignement de l'histoire naturelle qui, depuis Bourguet, c'est-à-dire depuis un siècle, était resté lettre morte.

C'est alors que Louis Coulon, - dont plusieurs d'entre vous se rappellent sans doute encore la sympathique figure vint suppléer à cette lacune en cherchant à obtenir une place au soleil pour la science qui lui était chère. Formé par de bonnies études à Paris, éclairé par le contact des savants naturalistes qui brillaient en France à cette époque, il sentait mieux que persónne ce qui nous manquait pour nous mettre en état de prendre notre part des recherches organisées dans tous les pays civilisés. Mais pour y parvenir, il fallait trouver un homme animé des mêmes intentions et de la même ardeur que lui, un professeur capable et surtout assez désintéressé pour se contenter des conditions modestes qu'on pouvait lui offrir. Grâce à l'initiative de Coulon, un jeune savant du plus brillant avenir allait renouer la tradition créée par l'auteur du Traité des pétrifications et devenir le chef et l'âme du mouvement scientifique qui illustra Neuchàtel il y a déjà plus d'un demi-siècle.

Le nom de L. Coulon est aujourd'hui inséparable de celui d'Agassiz. C'est à lui que nous devons l'établissement de ce naturaliste à Neuchâtel. C'est lui qui sut découvrir et encourager ce génie naissant, en lui fournissant les moyens de mettre en lumière ses talents de professeur et en lui procurant, pendant la plus belle période de cette vie laborieuse, à cette époque de jeunesse ardente et enthousiaste, un asile tranquille pour élaborer et publier les multiples travaux qui ont à juste titre fondé sa réputation. 
Jean-Louis-Rodolphe Agassiz naquit le 28 mai i 807 , dans la maison de cure de Môtier en Vully. Le voisinage de nos lacs, la société des pêcheurs el des chasseur's, nombreux dans la région, n’ont pas été sans exercer une influence sur la carrière de ce jeune homme qui, de bonne heure déjà, manifeste un goût très vif pour l'étude des choses de la nature et dont le choix des plaisirs montrait déjà le sens de l'observation. Au reste, tout dans l'éducation du futur naturaliste semble aroir concouru à préparer cette merveilleuse carrière de travaux et de découvertes.

Il commença en I8I 8 ses études classiques au collège de Bienne, puis les continua ensuite à l'Académie de Lausanne, où il se trouva en relations avec le professeur D.-A. Chavannes, directeur du Musée cantonal et possesseur d'une superbe collection d'histoire naturelle. Il en profita largement et fit tant de progrès dans l'étude de la zoologie et de l'anatomie comparée, que son oncle, le $\mathrm{D}^{\mathrm{r}}$ Matthias Mayor, le fameux chirurgien, se dit qu'il fallait en faire un médecin. C'est ainsi qu'il se trouva lancé dans la carrière des professions libérales où il devait se tracer lui-même un chemin tout autre qu'on ne l'eùt pensé. Aussi le jour où il dit son dernier adieu à l'établissement dans lequel il venait d'achever son éducation première, la pensée de l'investigation scientifique envahit son esprit. Il obtient la permission d'étudier la médecine et, en r824, à l'âge de dix-sept ans, il entre à l'Université de Zurich, où il séjourne pendant deux ans, puis il se rend à Heidelberg pendant une année et arrire enfin à Munich en 1827 .

A ce moment déjà ses goûts l'attiraient irrésistiblement du côté des sciences naturelles ét il éveille les plus belles espérances. Il se fait remarquer au milieu de ses condisciples et devient le centre d'un club d'instruction mutuelle et de discussion, qu'on surnommait alors la "petite Académie des sciences", et auquel assistaient souvent les professeurs. Avec sa vive ima- 
gination, son enthousiasme et sa facilité de parole, il était la cheville ouvrière de l'association. C'est ainsi qu'en se jouant il faisait son apprentissage de professeur. En 1829 , il subit à Erlangen ses examens de docteur en philosophie et l'année suivante, à Munich, ceux de docteur en médecine.

Pendant son séjour à Heidelberg ses pensées se trouvèrent ramenées sur un sujet caressé dès son enfance, lorsqu'il vivait au milieu des pêcheurs du lac de Morat, et c'est alors qu'il conçut le projet de son ouvrage sur les poissons d'eau douce. Sa passion pour ces animaux et ses comnaissances sur ce sujet étaient connues; lors de son séjour à Munich, il lui fut fait à ce propos une proposition très honorable. Les voyageurs Spix et Martius étaient revenus depuis peu d'une expédition au Brésil et Spix était mort n'ayant qu'ébauché la description des poissons qu'ils avaient rapportés. C'est alors que Martius, ayant appris qu'Agassiz était préparé par ses propres études à traiter un sujet de cette nature, lui demanda de mener à bonne fin le travail commencé par son camarade. Le jeune étudiant - il n'avait que 2 I ans accepta avec empressement une offre qui lui permettait d'étendre ses connaissances sur la branche d'études qu'il affectionnait spécialement, et se tira avec honneur de cette tàche difficile, qui devait créer sa réputation. Le résultat de ses études, entreprise extraordinaire qu'il avait tenue secrète et dont il voulait faire la surprise à son père, fut un volume accompagné de 97 planches, la plupart coloriées, qui attira immédiatement sur lui l'attention du.monde scientifique (r829). Son début est beau et il saura le mettre à profit pour un grand dessein. Engagé dès lors dans une voie féconde, il ne rencontre que des encouragements.

A son retour en Suisse, ses parents, qui avaient déjà fait pour lui des sacrifices considérables en raison de leur situalion de fortune, étaient impatients de lui voir commencer sa carrière pratique. Aussi ce ne fut pas sans peine qu'il obtint 
d'eux la faveur d'aller faire un séjour à Paris. Dès son arrivée dans la capitale, en I83 I, Agassiz s'empressa de se rendre auprès du grand naturaliste Cuvier, qui le reçoit avec la plus extrème bienveillance.

Distinguant chez le jeune savant les preuves d'un réel mérite et ayant pu apprécier la valeur de ses premiers travaux, il mit à sa disposition, avec une libéralité aussi rare que remarquable, tous les matériaux que lui-même avait réunis pour une histoire des poissons fossiles, renonçant à s'en servir pour enrichir l'œuvre de son jeune protégé. Cet héritage ne pouvait tomber en de meilleures mains et un pareil acte de désintéressement scientifique honorait autant celui qui en avait eu la pensée que celui qui en était l'objet.

Cuvier venait de créer la paléontologie et de révéler l'importance de cette nouvelle science-; il arait fait connaître de grandes choses en créant une science nouvelle. Agassiz de son côté comprit tout de suite qu'elle était un complément indispensable de la zoologie et il profite des circonstances où il se trouve pour commencer à réunir les matériaux de sa principale publication, résultat de ses premiers pas dans les recherches paléontologiques. La connaissance qu'il fit de Cuvier fut le point de départ de sa vocation. La proposition du grand naturaliste était séduisante, aussi ce puissant encouragement acheva-t-il de décider sa carrière. Agassiz avait compris qu'il touchait à un moment critique de sa vie et qu'il était temps de prendre une décision définitive. Confiant dans son étoile, il prend bravement son parti, il sera naturaliste. Hélas! si ce n'était pas pour lui le chemin de la fortune, c'était au moins celui plus glorieux de la renommée. C'est dès cette époque que les relations les plus intimes s'établirent entre Cuvier et Agassiz. C'est aussi à partir de ce séjour à Paris qu'Alexandre de Humboldt, qui s'y trouvait et protégeait volontiers les jeunes gens studieux, lui témoigna le plus vif intérêt et resta dès lors son protecteur, son conseiller et 
son ami. La bienveillance de l'illustre voyageur, qui lui donna du reste la preuve de son amitié en lui fournissant les moyens de prolonger son séjour à Paris, ne devait plus lui laire défaut et exerça plus tard une grande influence sur sa carrière.

A la mort de Cuvier, en mai r 832, il s'agissait de terminer l'ouvrage (Histoire naturelle des poissons) que le grrand savant laissait inachevé. Valenciennes lui proposa de s'associer à ce travail en lui faisant des propositions fort engageantes. Mais la nostalgie du pays natal l'emporte el il refuse les onvertures qui lui sont faites. Il est trop peu Français de caractère et désire trop vivement s'établir en Suisse pour préférer la place qui lui est offerte.

Le jeune Vaudois, qui sortait des universités allemandes avec ses diplòmes de docteur en philosophie et en médecine, et des travaux qui avaient déjà attiré sur lui l'attention du monde savant, ne savait trop que faire. C'était beau sans doute d'avoir conquis l'estime des grands naturalistes, mais cela ne pouvait suffire. Son canton d'origine ne lui offrait aucun avenir. Ses parents ne pouvaient continuer à subvenir à son entretien et à ses dépenses, et désiraient le voir s'établir comme médecin pratiquant, n’importe où, pourvu qu'il pût gagner sa vie.

Mais son goùt et les aspirations de son génie le poussaient ailleurs que du còté de la médecine; ils l'entrainaient vers la carrière scientifique où il entrevoyait de vastes champs d'étude encore inexplorés. Pour cela il lui fallait des loisirs, des encouragements, surtout de l'argent, nerf aussi bien de la science que de la guerre, ou tout au moins une situation rétribuée qui lui procurât son pain quotidien. La nécessité d'une position lucrative devenait impérieuse.

Le jeune homme caressait l'idée - suivant en cela les désirs de sa mère - de venir s'établir à Neuchàtel, où il avait des parents, et se trouverait plus rapproché de sa famille, qui était venue habiter Concise. C'est alors qu'il s'adressa à Louis 
Coulon, l'âme de tout ce qui se faisait alors chez nous dans le domaine des sciences naturelles, lui exprimant le désir d'obtenir une place de professeur au collège, où depuis Bourguet personne ne s'était jamais livré à un enseignement de ce genre. Seul, en effet, Coulon pouvait lui tendre la main pour le tirer d'embarras et lui aider à prendre dans le monde la situation qu'il ambitionnait.

Ce fut pour Coulon un beau moment de sa vie que celui où il reçut la demande d'Agassiz, datée de Paris, 27 mars r 832. J'extrais de cette lettre les lignes suivantes: "Lorsque dans le courant de l'été passé j’ai eu le plaisir de vous voir, je vous ai exprimé à plusieurs reprises le vif désir que j'aurais de pouvoir me fixer auprès de vous et mon intention de faire des démarches pour chercher à obtenir la chaire d'histoire naturelle que vous fonderez dans votre lycée. Maintenant les choses doivent être plus avancées que l'an passé et vous m'obligeriez infiniment si vous pouviez bientòt me donner quelques renseignements là-dessus. J'ai communiqué mes projets à M. de Humboldt, que je vois fréquemment, et qui veut bien me tẹmoigner de l'intérêt en m'aidant de ses bons conseils; il pense que dans de pareilles circonstances il faut, surtout dans ma position, prendre ses mesures à l'avance. " Et plus loin il ajoute en terminant: "Vous me rendriez donc le plus grand service si vous vouliez me donner là-dessus vos directions et surtout mé dire de qui dépend la nomination de la chaire d'histoire naturelle, etc. »

Coulon encourage Agassiz en lui disant que la création à Neuchâtel d'une chaire d'histoire naturelle n'est pas chose impossible et lui demande s'il se contenterait de 70 à 80 louis par an pour ro heures de leçons par semaine, qui se donneraient dans les classes supérieures du collège, appelées alors auditoires de Belles-Lettres et de philosophie. Il ajoute qu'il ne peut pas attendre le moment de le voir et de l'avoir pour collègue. 8o louis! ce n'était pas très lucratif, mais Agassiz 
ne recherchait pas la fortune. Il accepte avec reconnaissance cet humble salaire qui fait rire aujourd'hui, car c'était son salut.

Après le sacrifice offert aux divinités de la science, qui faisaient invasion dans le sanctuaire classique - la création des deux chaires de mathématiques et de physique et chimie avait été, ainsi que nous venons de le voir, décidée quelques années auparavant - l'administration de la Bourgeoisie croyait avoir fait le nécessaire et hésitait, craignant de compromeltre l'avenir. Un des motifs de refus était, paraît-il, un déficit de fr. I4, ooo dans les finances de la ville, causé par la construction du Gymnase et les événements politiques de l'année précédente. Or ce déficit paralysait les courages et on se souciait assez peu de poser les bases d'un enseignement nouveau dont on ne sentait pas la nécessité.

Il fallut l'intervention presque hérö̈que de Louis Coulon pour aplanir les difficultés que rencontrait la création de cette nouvelle chaire d'histoire naturelle. Mais Coulon avait trouvé dans son protégé l'homme qu'il cherchait tant; il avait deviné en lui le génie capable d'éveiller dans notre pays, par sa parole ardente et son activité, l'amour des sciences de la nature et eût fait l'impossible pour l'avoir à ses côtés et faciliter l'essor de cette brillante intelligence, si remplie de promesses. Se plaçant lui-même en tête d'une liste de souscriptions qu'il colporta de porte en porte chez quelques personnes bénévoles, avec cette intrépidité calme, mais irrésistible de l'homme convaincu, il fut bientôt en mesure d'offrir un modeste traitement annuel de fr. 2000, garanti pendant trois ans, à celui qui ne devait pas tarder à illustrer Neuchâtel.

Le 18 juin, le Conseil de Ville autorisait l'érection d'une chaire de professeur d'histoire naturelle au Collège et décidait - sans doute d'autant plus facilement qu'il savait n'avoir pas à intervenir - que si la souscription ouverte dans ce but n'atteignait pas la somme voulue, la Caisse publique y pourvoirait. Voici ce qu'on lit à ce propos dans.les Ma- 
nuels du Conseil gुénéral : "Le Conseil a reçu communication d'une souscription ouverte en cette ville dans le but de pourvoir à la pension d'un professeur d'histoire naturelle, en attendant que les ressources de la Caisse publique permettent au Conseil de le salarier; cette souscription s'élevant déjà, quoique non encore bouclée, à environ 68 louis sur la somme de 8 o louis par an nécessaire à son objet et les souscripteurs s'engageant chacun pour sa part à la renouveler pendant trois ans, moyennant que la chaire soit incontinent établie, afin de profiter d'un professeur habile (M. Agassiz) quì est disposé à l'occuper, délibéré, le Conseil se déclare disposé à seconder les vues utiles qui lui sont communiquées et qui sont appuyées de la recommandation de la Commission d'éducation, et en conséquence il autorise l'érection d'une chaire de professeur d'histoire naturelle pour faire partie des établissements d'éducation en cette ville, avec garantie pendant trois ans d'un traitement annuel de 80 louis au professeur qui $y$ est appelé, en sorte que si la souscription ouverte dans ce but n'atteint pas la dite somme la Caisse publique y pourvoira. ")

Grâce à cet homme d'action, qui pendant de. trop courtes années porta chez nous si haut élevé l'étendard de la science, un souffle nouveau se fit sentir et Neuchâtel devint un véritable foyer scientifique. Agassiz prêchait par l'exemple. Semblable, ainsi qu'on l'a dit, à un aimant, il attirait les esprits d'élite qui devaient marcher sur ses traces. Il étail comme un brasier qui réchauffait jusqu'aux plus froids et aux plus indifférents. L'énergie qu'il déploya pendant ces quelques années fut quelque chose d'inouï et dont, ainsi que s'exprime un de ses biographes, l'histoire de la science n'offre peut-être pas d'autre exemple. Aussi on comprend qu'un tel homme ait donné une impulsion toute nouvelle à l'étude des sciences à Neuchâtel. Sans contredit, son séjour chez nous a créé un développement dont nous subissons aujourd'hui encore l'heureuse influence. 
Enchanté d'avoir enfin trouvé une situation, d'avoir un poste fixe que son imagination lui représente comme une fortune, Agassiz arrive pour en prendre possession. Il inaugurait une vocation qui devait laire le bonheur de sa vie. Enseigner fut, en effet, pour lui une passion. C'est ce qui explique l'influence extraordinaire qu'il exerça dans la suite sur ses élèves.

Mais tout manquait à Neuchâtel, salle de cours et collections. Il n'y avait aucun local disponible pour l'enseignement nouveau qu'il-s'agissait d'inaugurer. On eut recours à une salle de l'Hòtel-de-Ville, la salle actuelle de la Justice de paix, comme auditoire, et on aménagea un musée provisoire dans la maison voisine des Orphelins, PHôtel municipal, où on logea comme on put les collections que le nouveau professeur apportait avec lui et qu’il avait formées pendant ses études. Ces collections furent acquises l'année suivante pour le Musée d'histoire naturelle, alors en formation, pour le prix de I 2,00o francs, grâce au généreux concours de Frédéric-Guillaume III, du comte Louis de Pourtalès et de la Ville, ce qui fournit au jeune savant les premières ressources nécessaires à la publication de ses multiples travaux.

Les cours commençèrent en automne et Agassiz prononça sa leçon d'ouverture le I 2 novembre I832, en présence d'un nombreux public, sur les relations entre les différentes branches de l'histoire naturelle et les tendances actuelles de toutes les sciences. Son père assistait tout ému à cette séance, qui ouvrait à son fils sa carrière définitive. Son succès fut grand et le jeune professeur séduisit dès cette première leçon son nombreux auditoire. Grand, bien fait, possédant une figure aimable et un regard brillant d'intelligence, il gagnait la sympathie de tous ceux qui l'approchaient. Sa physionomie était franche et ouverte, son caractère attachant. Il y avait dans son enseignement, comme dans sa conversation, quelque chose de chaleureux, de communicatif, de familier et d'élevé. 
Il savait adapter son langage à l'état d'esprit de ceux qui l'écoutaient. Un entrain que rien ne pouvait contenir s'unissait chez lui à la facilité et au charme de la diction. Toujours prèt à créer des théories, à les discuter, à exposer des idées nouvelles, il captivait ses auditeurs par la verve et la clarté de son exposition. Il sut éveiller dans l'esprit de ses étudiants des besoins intellectuels élevés et leur communiquer cette soif de connaitre, cette ardeur au travail, cet amour du bien et de la vérité, qui furent la passion de toute sa vie. Aussi ses anciens élèves - qui malheureusement deviennent de moins en moins nombreux - parlent-ils d'Agassiz avec enthousiasme, de ses leçons captivantes et des courses scientifiques qu'il organisait.

Le professeur suppléait, en effet, au manque de matériel d'enseignement par des excursions qu'il faisait avec ses étudiants aux environs de Neuchâtel, au Mail, alors à l'état de forèt pittoresque, riche en plantes de toutes espèces, dans les Gorges du Seyon, solitude sauvage où ne passait qu'un étroit sentier très pittoresque, dans les forêts de Chaumont, au Creux du Vent, excursions dans lesquelles il initiait ses élèves à la pratique de l'histoire naturelle. Ces courses, en vue desquelles il avait publié un petit opuscule : Tablean synoptique des principales familles naturelles des plantes (1833), étaient des fètes pour les étudiants, qui voyaient dans leur maître un compagnon alerte, plein d'entrain, de vigueur, de gaieté, et dont toute la personne éveillait en eux le feu sacré de la science.

A còté de ses leçons et afin d'associer le public à son activité, le nouveau professeur donne des cours publics et des conférences dont le produit est appliqué à l'agraindissement du Musée. Gràce à l'autorité de sa parole, au charme de sa voix et de sa figure, il passionnait ses auditeurs qui restaient suspendus à ses lèvres, même lorsqu'il traitait les sujets les plus abstraits, Aussi les questions qui préoccupaient les naAGiASSIZ. -2 
turalistes pénétraient-clles, assure-1-on, jusque dans les sàlons. Ce talent de parole, qu'Agassiz possédait à un haut degré, fut un de ses principaux moyens d'action et contribua grandement à sa célébrité.

Ce n'est qu'en r 835 que les Conseils de la Bourgeoisie décrétèrent la fondation d'une chaire d'histoire naturelle el qu'Agassiz, de professeur libre qu'il était aupararant, devint professeur régulier. On lit à ce sujet dans les Manuels du Conseil général, à la date du 27 avril, ce qui suit: "Entendu un rapport de la Commission d'éducation touchant la chaire d'histoire naturelle dont l'établissement n'a eu lieu en 1832 que sur un pied provisoire, à mesure que les honoraires du professeur ont été procurés en presque totalité par des souscriptions pour le terme de trois ans, ce rapport concluant : $I^{\circ}$ à ce que la chaire de professeur d'histoire naturelle soil décrétée d'une manière permanente, vu son utilité reconmue surtout par le zèle et les vastes connaissances de M. le professeur Agassiz, $2^{\circ}$ à ce que la Ville prenne à sa charge la totalité du paiement des honorairés de ce poste, qui ont été fixés à 80 louis par an, $3^{\circ}$ enfin à ce que le professeur puisse être autorisé à prendre des congés ou vacances de quatre à cinq mois durant l'année pour faire des courses ou royages, et, considérant l'importance de conserver le célèbre professeur que cet établissement a le bonheur de posséder, le Conseil fixe à roo louis par an, à dater du premier juillet prochain, les honoraires de mon dil sieur Agassiz. ")

Neuchâtel et non plus l'initiative privée offrait ainsi définitivement au jeune savant la position stable qu'il ambitionnait et dont il avait besoin pour se livrer à ses études de prédilection.

Agassiz n'était établi à Neuchâtel que depuis cinq ans lor'sque des appels lui furent successivement adressés de Genève et de Lausanne, où on nous enviait sa puissante personnalité, ce professeur plein d'enthousiasme, ce travailleur prodigieux. 
Lorsqu'on apprit qu'il venait de décliner les offres engageantes qui lui étaient parvenues, on chercha à lui témoigner le gré qu’on lui savait de sa fidélité. La Ville lui témoigna sa joyeuse satisfaction en l'honorant d'une distinction qui le rattachait plus intimément au pays. Elle le nomma bourgeois de Neuchâtel, confirmant en cela une décision du Conseil d'État, qui lui avait déjà conféré, quatre ans auparavant, la nationalité neuchàteloise. D'un autre côté, cinquante souscripteurs de bonne volonté s'engageaient à lui fournir, pendant une période de six années, une somme annuelle de 2000 francs pour lui procurer les moyens de développer de plus en plus ses recherches et ses travaux.

Voici la lettre de remerciements qu’il envoya à ses généreux donateurs. Adressée à MM. Terrisse, président de la Commission d'éducation, et Louis Coulon, directeur du Musée, elle explique les circonstances dans lesquelles il s'était trouvé avant son arrivée à Neuchâtel et montre l'attachement qu'il avait pour notre ville.

\section{Messieurs,}

Jeudi 22 mars 1838.

"Il y a maintenant sept ans que je me trouvais en Suisse, sur le point d'être obligé d'abandonner l'étude des sciences naturelles, à laquelle j'avais consacré déjà plusieurs années de ma vie, pour embrasser une carrière plus assurée, ne pouvant prolonger les sacrifices que l'on avait faits pour moi jusqu'alors. Je devais aller à Paris terminer des études de médecine et revenir bientôt pratiquer dans mon pays cet art dont l'apprentissage m’avait initié aux délices de l'étude de la nature, en même temps que j’apprenais à connaître toutes les misères auxquelles l'homme est exposé sur la terre. Je nourrissais encore l'espoir que quelque incident heureux me ramènerait à mes travaux de prédilection. Ce fut alors que je fis la connaissance de Messieurs Coulon ${ }^{1}$; je savais qu’ils culti-

1. Louis Coulon r 804-1894 et son père Paul-Louis-Auguste Coulon 1777I 855 . 
vaient l'un et l'autre diverses branches de l'histoire naturelle avec une persévérance et un zèle que l'on rencontre rarement, mème chez ceux qui en font leur état. La bienveillance qu'ils me témoignèrent me rendit le courage qui m'avait soutenu à travers toutes les difficultés que j'avais eues à surmonter, mais qui était près de m'abandonner. J'aime maintenant à me rappeler ces circonstances et à les exprimer parce qu'elles ont eu une influence marquée sur ma vie. En effet, l'accueil que me firent à Paris les deux hommes qui ont eu le plus d'influence sur le développement de la science, depuis qu'elle est sortie de l'oubli auquel la prépondérance d'autres intérèts l'avait réduite 1 , me donna assez de confiance dans mes propres forces pour me faire abandonner une carrière qui devait ètre mon gagne-pain et poursuivre les chances d'une vie dont je ne connaissais encore que les douceurs. Ma résolution était prise, j’avais renoncé à la médecine pour devenir naturaliste. De ce moment, comme si ma persévérance devait être mise aux plus rudes épreuves, j'eus à lutter contre les privations les plus dures et ce n'est qu'à l'amitié de M. de Humboldt que j'ai dù les moyens d'avoir pu les braver au moment où je croyais devoir y succomber. Et ce que j’étais loin d'attendre, c'est qu'en même temps mes amis de Neuchâtel se souvinrent de moi et eurent assez de confiance dans mon avenir pour m'offrir une chaire et les moyens de vivre honorablement au milieu d'eux. J'embrassai avec reconnaissance cette proposition qui me procurait les facilités nécessaires pour me livrer tout entier à une étude qui me semblait ma vocation. Vous savez, Messieurs, tout ce que la ville, ses magistrats, ses habitants, l'auguste souverain de notre pays, ont fait pour moi dans si peu de temps, pour moi qui avais si peu de titres à des marques aussi éclatantes de tant de bienveillance, et si je ne vous le rappelle pas plus spécialement, c'est par la crainte de blesser ce sentiment intime de bienséance que possèdent à un si haut degré les Neuchâtelois. Mais ce que je ne puis taire, c'est que c'est à ces avantages que j'ai dû d'avoir pu mettre

1 Georges Cuvier 1 $769-1832$ et Alexandre de Humboldt $1769-1859$. 
au jour les recherches dont je m'étais occupé sans relàche depuis que la science a fait ma seule occupation. La nouvelle marque d'affection, de distinction, je dirai même de considération que vous venez de me donner en votre nom el celui de vos compatriotes, me permet de vous dire que si la réputation qu'ont acquise ces travaux avail pu vous faire craindre de me voir quitter Neuchâtel, vous auriez méconnu une partie des motifs qui m'y attachent. Car outre la reconnaissance que je dois à tous ceux qui m’ont aidé à devenir ce que je puis être, il est une raison bien puissante qui m'attache à cette localité, c'est la conviction que j’ai acquise que la science y est aimée, favorisée, qu'elle est le point d'appui de toutes les institutions du pays, qu'elle y est entourée de considération, qu'on lui élève des monuments presque gigantesques et qui seraient disproportionnés avec son étendue si la générosité des citoyens ne venait au-devant de leurs besoins et n'en assurait ainsi la prospérité. Cette conviction, le désir de virre en dehors de toutes les tracasseries des coteries d'une grande ville et l'affection que j'ai vouée à ceux qui ont assez de foi en moi pour m'aider à-devenir utile à la science que j'ai embrassée, sont des motifs trop puissants pour qu'ils n'aient pas aisément balancé des avantages purement matériels qui m'étaient offerts pour m'attirer ailleurs. i)

Agassiz était venu s'établir à Neuchâtel au milieu de circonstances difficiles. C'était à la fin d'une époque de luttes politiques et de révolution. Cependant l'orage se calma et les esprits cultivés sentirent le besoin de rompre avec les préoccupations du moment, qui divisaient profondément le pays. Les hommes de science, las de dissensions pénibles, cherchèrent un terrain neutre sur lequel ils pussent se rẻunir et s'entendre. D'accord avec Louis Coulon, il sentit le besoin de faciliter l'élan qui se dessinait vers un progrès réel dans le domaine intellectuel, en groupant ensemble les hommes isolés qui s'intéressaient plus spécialement aux progrès des sciences. II employa son influence et son activité à réunir en un faisceau 
les forces éparses dans notre ville, voulant reproduire à Neuchâtel ce qu'il avait créé pendant le cours de ses études à Munich, des rendez-vous de personnes laborieuses, qui désiraient, mettre en commun leurs connaissances scientifiques.

C'est dans ce but que fut fondée, le 6 décembre 1832, la "Société des sciences naturelles. " Ainsi se trouvait formée, sur la base du principe fécond de l'enseignement mutuel et du libre échange de vue personnelles, un centre de culture intellectuelle et de libre discussion où tant de personnes ont apporté le tribut de leurs recherches, les primeurs de leurs découvertes, cela en vue de répandre chez nous la connaissance des données scientifiques. Il est inutile de faire ressortir l'essor que prit cette Société el quel éclat les travaux d'Agassiz firent rejaillir sur elle et sur la ville qu'il habitait. La collection des quatre tomes de ses Mémoires et des trente volumes de son Bulletin est la meilleure preuve de la vitalité qu'elle a jusqu'ici montrée, et forme comme un monument durable de son activité.

Si le nouveau professeur se préoccupait des besoins de son enseignement, il s’inquiétait davantage encore de ses études personnelles et de la publication de leurs résultats. Il avait l'esprit trop vaste et trop remuant pour s'enfermer dans une spécialité et se jetait résolument dans les plus vastes entreprises, comme s'il avait senti ses forces inépuisables. Un des traits distinctifs de son caractère était, en effet, une curiosité passionnée qui le poussait à tout; à cette curiosité toujours active s'ajoutaient une mémoire dont l'étendue tenait du prodige et une facilité singulière de passer d'un travail à un autre, immédiatement, sans effort, faculté qui peut être a contribué plus que toute autre à multiplier son temps et ses forces. Agassiz était homme à ne tenir compte ni de l'argent, ni du temps, ni de la fatigue, lorsque la science était en jeu, et pendant l'exécution même d'un ouvrage original il n'hésitait pas à s'engager dans de nouvelles investigations, telle était la 
capacité intellectuelle de ce naturaliste, telle était sa puissance de travail, que ces recherches si diverses et si nombreuses, loin de produire dans son esprit une confusion inextricable, semblaient toutes hii ouvrir des voies nouvelles pour atteindre aux vérités naturelles. Les quatorze années qu'il passa à Neuchâtel furent tout particulièrement des années de recherches et de publications, et on a peine à concevoir qu'un seul homme, mème avec des aides capables, ait pu, dans celte courte période, produire une somme de travail si énorme.

A Neuchâtel, le jeune savant n'a plus, comme à Munich et à Paris, des collections importantes à consulter, des quantités de matériaux à utiliser ; il n'a pas non plus des sarants prêts à venir à son aide. Mais il est le premier, le chef, et il rallie autour de lui un groupe de personnes qui rappellent sur un théàtre plus modeste la petite Académie de Munich. Avec la confiance d'un homme pourvu des ressources de la puissance et de la fortune, il se -mit à réunir autour de lui tous les éléments d'un centre scientifique.

Il était arrivé à Neuchâtel accompagné de deux dessinateurs, qui préparaient les planches de ses nombreux ouvrages ; il leur en adjoignit bientôt un troisième, M. Burckhardt, qui l'a accompagné aux États-Unis et l'a suivi dans toutes ses expéditions. Il fallait encore à l'infatigable travailleur un mouleur. Ce fut M. Stahl, artiste habile, employé plus tard au Muséum d'histoire naturelle de Paris, qui reproduisit en plàtre les moules de coquilles et d'échinides, ou les fossiles rares dont il était fait des collections pour les échanger ou les vendre aux musées d'Europe. Et ce n'était pas tout. Les divers travaux qu'il s'agissait de publier exigeaient le concours d'un lithographe, organisé de manière à reproduire les desseins des artistes dont le savant s'était entouré. Ce fut Hercule Nicolet, qui se chargea de reproduire les planches destinées à illustrer ces travaux, planches qui passaient alors pour les plus beaux spécimens de la lithographie à ses débuts. 
A cette phalange d'artistes s'en joignait une autre, d'un caractère scientifique, car à mesure que le champ des études du professeur s'élargissait, le travail d'observation et de recherches devenait plus considérable. Agassiz cherchait un secrétaire capable de l'aider dans ses travaux. Il le troura à Berne, en la personne d'Édouard. Desor, jeune homme plein d'entrain et d'ardeur, aimant le travail, ayant soif d'activité. Son'esprit ouvert, sa vive intelligence, lui permirent de se familiariser rapidement avec les différentes branches des sciences dont il était appelé à s'occuper. Deux ans après, Charles Vogt, qui venait de passer ses examens de médecine, jeune savant, remarquable par sa sagacité et la pénétration de son esprit, vint le rejoindre. Initié aux aspirations du maître, il ne tarda pas à prendre une part active à ses travaux. Tous deux étaient jeunes et forts, possédaient la gaieté, l'intelligence, la soif de connaître; ils avaient le feu sacrẻ qui fait affronter tous les obstacles.

Agassiz avait déjà conçu, pendant ses études, le plan d'un grand ouvrage sur les poissons d'eau douce. C'est le premier dont il se soit sérieusement occupé, et celui peut-être qui a été le plus constamment le but des travaux et des efforts des premières années de sa vie scientifique. Cet onvrage, qui est intitulé Histoire naturelle des poissons d'eau douce de l'Europe centrale, et qui avait été entrepris sur un plan très vaste, est resté malheureusement inachevé. De 1839 à r 845 , il en a paru trois livraisons, publiées en collaboration avec Ch. Vogt et accompagnées de 55 planches folio, la plupart coloriées. Elles traitent de l'embryologie et de l'anatomie des Salmonides.

Mais Agassiz n'avait pas tardé, sur les conseils de Curier, à étendre ses recherches des poissons vivants aux poissons fossiles, et alors s'ouvrit devant lui ce vaste champ dans lequel il devait recueillir une si riche moisson. Les Recherches sur les poissons fossiles sont aussi une de ses premières con- 
ceptions. Cette volumineuse publication, qui comprend cinq volumes de texte et un atlas de près de 400 planches, commencée en 1833 , ne fut achevée qu'en 1843 , avec l'appui de la Société géologique de Londres et de généreux protecteurs.

Ce bel ouvrage, qui peut être regardé comme la continuation des "Recherches sur les ossements fossiles ", de Cuvier, lui valut des distinctions flatteuses de diverses Académies et Sociétés, et les applaudissements des savants les plus distingués. C'est dans cette œuvre, qui reste un des principaux monuments de sa gloire, que brillent surtout les qualités éminentes du savant paléontologiste et que sa riche imagination prend tout son essor en se laissant cependant toujours guider par une critique sage et raisonnée, basée sur un travail consciencieux et sur une analyse minutieuse des plus petites parties de l'organisme.

La poursuite de la besogne entreprise exigeait des visites dans les Musées et les collections particulières. Agassiz dut faire de fréquents voyages, tour à tour en France, en Allemagne, en Angleterre, en Écosse et en Irlande. Partout on se montrait ravi de recevoir le jeune savant. "Mon voyage, écrit-il d'Édimbourg à Aug. de Montmollin, le ı3 septembre I834, a été une fète continuelle, partout j’ai été reçu avec la plus grande distinction et accueilli avec empressement. Les matériaux pour mon ouvrage m'arrivent comme la pluie et les brouillards du pays. A Édimbourg, j'ai eu un triomphe inattendu dans la distinction que l'on m'a accordée au moment où je m'y attendais le moins. Dans une séance publique, en présence de plus de 2000 personnes, M. Buckland a fait un grand étalage de mes travaux et m'a proclamé le premier naturaliste actuel, et son jugement a été accueilli par une acclamation générale et des applaudissements sans fin. Vous pouvez penser que cela n'est pas sans avantage pour le succès de mon ouvrage, d'où dépend aussi mon bien être matériel. „ La Monographie des poissons fossiles du vieux grès 
ronge ou système dévonien, etc., accompagnée d'un atlas folio de 43 planches, faite à la demande de l'Association britannique pour l'avancement des sciences, vint compléter en I 844-45 la publication la plus importante qu'Agassiz ait créée pendant son séjour à Neuchâtel.

Mais la prodigieuse activité de cet homme ne pouvait être satisfaite par un seul objet d'études. Il avait l'eśprit trop vaste, il était trop entreprenant pour réserver son attention à une seule classe du règne animal. Malgré l'incroyable labeur qu'exigeaient ses études sur les poissons, il s'occupa aussi des mollusques, qui furent de sa part l'objet d'études neuves et originales. La comparaison des coquilles fossiles avec les coquilles vivantes occupa premièrement son esprit. Il publia ainsi tout d'abord un Mémoire sur les moules des Mollusques vivants et fossiles (г 839 ), auquel succédèrent des Études critiques sur les Mollusques fossiles (1840-45) et une Iconographie des coquilles tertiaires (I 845). Les Échinodermes firent de sa part et de celle de Desor l'objet de travaux importants. Il trouve dans les fossiles des marnes et calcaires jaunes de Neuchâtel, qu'Aug. de Montmollin venait de décrire sous le nom de " terrain crétacé du Jura ", les matériaux d'une première étude sur les Échinodermes de cette époque ( 835 ), la plupart encore inconnus ; puis il public les Monographies d'Échinodermes vivants et fossiles (1838-42), la Description des Échinodermes fossiles de la Suisse (I839-40) et le Catalogue raisonné des Échinides (1847).

Agassiz semblait voué d'une manière presque exclusive aux recherches de zoologie et de paléontologie. Mais bientôt ces deux domaines ne lui suffisent plus, et un champ de recherches nouveau vient s'offrir à lui. Chacun connaît aujourd'hui la théorie glaciaire, chacun a entendu parler de l'ancienne extension des glaciers, bien loin au delà de leurs limites actuelles, des roches polies résultant de leur frottement, des blocs erratiques qu'ils ont transportés à de grandes distances 
et à de grandes hauteurs. Mais, avant i 837 , tout cela était ignoré ou tout au moins la question n'avait encore été soulevée et discutée que très discrètement.

Deux hommes dont les noms, malheureusement trop ignorés, restent attachés à celte grande découverte, Venetz, mis sur la voie par un vieux chasseur de chamois, Perraudin, et Jean de Charpentier, étaient seuls dépositaires d'une théorie fondée sur des observations irréfutables. Mais cette théorie était tellement en désaccord avec les idées reçues, elle était si hardie, que ces modestes sarants n'osaient pas la proclamer, parce qu'ils ne parvenaient pas à la faire prendre au sérieux. Elle rencontrait de nombreux incrédules, et parmi eux Agassiz ui-même. Charpentier lui fournit l'occasion de la vérifier, en l'invitant à passer ses vacances de I 836 auprès de lui. Enthousiasmé par ces découvertes, à la vue d'un champ nouveau d'investigation, converti par Charpentier lui-même aux idées nouvelles, il devient aussi ardent à les défendre qu'il l'avait été auparavant à les combattre.

Le 24 juillet 1837 , la Société helvétique des Sciences naturelles se réunissait pour la première fois à Neuchâtel. Pour la recevoir dignement, on avait à la hâte transporté dans le noureau bâtiment du Gymnase et aménagé dans les nourelles salles qui leur étaient réservées, les collections du Musée d'histoire naturelle, déjà fort accrues, qu'on avait installé provisoirement dans la Maison des Orphelins, ainsi que la quantité de matériaux de toute espèce, qui, faute de place, attendaient dans les combles de la maison Coulon le moment d'ètre mis sous les yeux du public. Agassiz, nommé président, profita de la circonstance pour développer devant cet auditoire d'élite la théorie glaciaire.

Son discours, conçu pendant la nuit qui précéda la réunion - tant était grande sa facilité de conception et de rédaction - et qui contenait des théories si extraordinaires pour l'époque, eut un retentissement considérable. Présenté avec l'au- 
torité d'une réputation et l'ascendant d'un grand enthousiasme, il ne pourait manquer de faire sensation. Les contradicteurs furent nombreux, car jusqu'alors les géologues avaient unanimement attribué à l'action de l'ead le transport des blocs erratiques et les roches polies et striées. Aussi on comprend leur fureur en présence des assertions hardies d'un jeune savant de trente ans, qui venait bouleverser les idées reçues. Agassiz s'attira les foudres de Léop. de Buch, les protestations d'Élie de Beaumont et les murmures de tous les partisans des anciennes doctrines. Les uns juraient par la glace, les autres par l'eau et les torrents. On était effrayé à Neuchàtel de l'altitude qu'avait prise le président de la Société helvétique êt de l'audace de ce jeune homme qui, en proclamant des idées opposées à celles des maîtres de la géologie, avait pour ainsi dire cassé les vitres et transporté dans la science la révolution à peine comprimée dans le domaine de la politique: Mais beaucoup d'excellents esprits riaient de ces terreurs ; ils sentaient une ère de joyeux épanouissement, de travail et de découvertes s'ouvrir devant leurs yeux, et ils saluaient l'aurore des jours glorieux qui allaient luire sur notre cité. Et ils avaient raison, car malgré de vives oppositions la théorie proclamée allait faire son chemin.

Cependant, le jeune savant ne se borne pas à discuter avec des paroles. Fournir des preuves, apporter la démonstration des faits qu'il avance, devient le seul souci du hardi novateur, qui n’était pas homme à reculer devant les fatigues d'une pareille tâche. En présence d'une théorie aussi nouvelle ef aussi hardie, la discussion devait nécessairement se porter sur les glaciers actuels, car pour admettre que les glaciers des Alpes aient pu s'avancer jusqu'au Jura, il fallait savoir en vertu de quelles lois ils se meurent dans leurs limites actuelles. C'est dans ce but qu'il entreprit pendant huit années consécutives, de i 838 à ı 845 , ces fameuses expéditions alpestres qui eurent dans le monde scientifique un si grand 
retentissement, expéditions poursuivies avec autant d'ardeur que de persévérance et d'intrépidité, et conduites arec la patience d'un bénédictin et l'exaltation d'un croisé.

Agassiz était accompagné dans ses campagnes du glacier de l'Aar (i 840 à I 845), les plus connues, de ses deux collaborateurs Desor et Vogt, auxquels vinrent s'adjoindre pendant quelque temps Célestin Nicolet, le pharmacien-naturaliste de la Chaux-de-Fonds, et deux étudiants, François de Pourtalès et Henri de Coulon. Pendant les premières années, une excavation sous un énorme bloc de gneiss de la moraine médiane servit d'abri à la petite troupe des explorateurs. Cette habitation, plus que primitive, devenue célèbre sous le nom pompeux d'Hôtel des Neuchâtelois, acquit bientòt, comme l'on sait, une telle célébrité qu'elle devint le rendezvous des savants nationaux et étrangers, sans compter les touristes et les curieux, attirés les uns par l'intérêt des études glaciaires, les autres par la réputation d'Agassiz et la singularité de ses observations.

C'est un curieux spectacle pour l'époque que celui de ces jeunes savants perdus dans la solitude, duns un désert aussi morne et silencieux que les régions polaires, au milieu d'un paysage triste mais grandiose, résolus à pénétrer au péril de leur vie les secrets de cette nature redoutable, et déployant dans leurs travaux une persévérance, une ardeur, quelquefois une hardiesse bien faites pour captiver l'attention des plus indifférents. Renonçant pour de longues semaines au bien-ètre de la vie civilisée, dans Jeur cabane au milieu des glaciers, où ne monte pas le bruit des plaisirs du monde et des affaires publiques, ils rêvent de pénétrer les plus intimes secrets de la nature.

Tandis qu'Agassiz et ses compatriotes s'établissaient sur le glacier de l'Aar, son ami et collègue Arn. Guyot, avec une abnégation fraternelle jamais démentie, lui venait en aide en étudiant les terrains erratiques semés par les anciens glaciers 
disparus au nord et au sud des Alpes, et y consacrait ses vacances pendant sept années consécutives. Ses recherches l'entraînèrent ainsi bien loin du centre d'activité de ses compagnons d'œuvre. C'est la raison pour laquelle on ne le voit pas faire partie du groupe des explorateurs. Néanmoins, ses études n'en faisaient pas moins partie du plan général, car tous les résultats obtenus étaient réciproquement communiqués et comparés. Même pendant ses excursions, Guyot adressait à Agassiz les observations qu'il faisait, lui soumettant ses doutes, faisant appel à son expérience.

L'intention d'Agassiz était de publier sur les glaciers un grand ouvrage en trois volumes. Le premier devait contenir le résultat de ses observations personnelles, le second les travaux de Guyot et dans le troisième Desor exposerait le phénomène erratique en dehors de la Suisse. Le premier volume seul fut publié en I847, sous le titre Nouvelles études et expériences sur les glaciérs actuels. Il renferme un résumé des observations faites pendant les séjours au glacier de l'Aar. Pendant qu'il était sous presse, son auteur partait pour les États-Unis et cette publication ne fut jamais reprise. Quelques années auparavant, en I840, Agassiz avait fait paraitre le résultat de ses premières observations dans les $\mathrm{Al}$ pes valaisannes, sous le titre Études sur les glaciers, ouvrage accompagné d'un atlas folio de 32 planches.

Cependant le savant professeur ne s'est pas toujours borné à la publication de travaux originaux qui montraient le génie scientifique de leur auteur. Il s'est aussi attaché à des recherches plus arides. L'esprit humain est ainsi fait qu'il se délasse d'un travail par un autre travail et qu'il cherche des contrastes dans les études les plus dissemblables. Il rédigea patiemment un ouvrage en quatre volumes, fruit de ses nombreuses lectures et de sa vaste érudition, qui sous le titre de Nomenclator zoologicus (I842-1846) renferme la liste de tous les noms de genre du règne animal avec l'indi- 
cation des auteurs, la mention des ouvrages où ils sont cités pour la première fois et la date de leur publication. Avec ce livre, qui nécessita des recherches infinies et le concours de plusieurs spécialistes, l'auteur a èu le mérite d'aroir exécuté une entreprise devant laquelle bien d'autres avaient reculé et qui semblait marquer la fin d'une grande période de ses travaux. En même temps, il travailla à une Bibliographie zoologique et géologique en quatre volumes, qui fut publiée après son départ pour l'Amérique (r848-I854). Si nous ajoutons à ces différentes publications d'ordre zoologique, paléontologique et géologique, un grand nombre de notices sur des points spéciaux d'histoire naturelle, parues dans des revues suisses, françaises, allemandes, anglaises, italiennes, russes, qui se succédèrent incessamment, on pourra se faire une idée de la somme de travail et d'énergie qu'a montré Agassiz pendant són séjour à Neuchàtel. Et avec cette besogne il trouva encore le temps de traduire en allemand la Géologie et Minéralogie, de Buckland (1839), et en français la Conchyliologie minéralogique de la Grande-Bretagne, de Sowerby (i 845 ).

Un de ses biographes a écrit que son établissement à Neuchâtel fut scientifiquement un succès unique pour l'histoire naturelle. "Le résultat de son séjour de quatorze ans, dit-il, a été la publication de plus de vingt volumes avec 2000 planches folio ou octavo et de beaucoup de brochures indépendantes, le tout très bièn écrit, admirablement imprimé et illustré avec profusion par des dessins extrêmement corrects, fait si estimable qu'il procura une juste célébrité non seulement à Agassiz, mais aussi à Neuchâtel qui était alors une petite ville de moins de 6000 habitants. Les Neuchâtelois peuvent être fiers d'une telle cuvre; leur grande libéralité pour la science et le cas qu'ils firent de la rare valeur d'Agassiz lui permirent de poursuivre avec une vigueur inaltérable ses remarquables recherches scientifiques renommées dans le monde entier." 
Jusqu'en I 840 à peu près, les hautes études avaient été entièrement à la charge de la Bourgeoisie. L'État comprit enfin qu'il ne pouvait plus rester étranger aux efforts faits dans l'intérèt de l'instruction générale et le 17 mars 1838 Frédéric-Guillaume III, répondant favorablement à la démarche faite auprès de lui, décidait la création de notre premier établissement d'instruction supérieure. Agassiz était nommé professeur le 26 juillet 1840 et le i 8 novembre de l'année suivante il prenait pour la première fois la parole, à l'occasion de l'inauguration de l'Académie naissante, dans celte salle même, et prononçait un discours sur la succession et le développement des êtres organisés à la surface du globe. Il fut un des principaux organes de cette Académie qu’il avait vu naìtre et qui, sans sa présence à Neuchâtel, ne se serait peut-être pas fondée ou tout au moins soutenue. L'année suivante il en était nommé recteur.

Lorsqu'au printemps de i 845 il annonça un dernier cours public de douze leçons sur le plan de la Création, son auditoire fut plus attentif que jamais. L'importance n'était pas seulement dans le sujet traité, mais dans la personne du professeur, qui charmait par sa parole toujours simple et facile, et dans cet enseignement final qui était pour lui, avant de quitter l'Europe, comme son testament scientifique. Son départ, l'année suivante, porta à l'Académie un coup sensible. Deux ans après éclatait la Révolution et notre premier établissement d'instruction supérieure, qui pendant sa courte existence avait marqué une trace si profonde dans. la vie intellectuelle du pays, se trouvait supprimé.

La période de travail incessante dans laquelle Agassiz s'était trouvé plongé pendant son séjour à Neuchâtel ne fut cependant pas pour lui une période heureuse. Il fut en proie à des soucis domestiques auxquels vinrent bientòt se joindre des embarras d'argent. Les expéditions aux glaciers et ses publications avaient été coûteuses. Il avait dù requérir le 
concours de deux aides, de trois dessinateurs, d'un mouleur, d'un lithographe. Le feu de son activité l'avait jusque là emporté sur la froide raison qui calcule et n'entreprend rien sans les facilités nécessaires. Il s'était peu inquiété de l'équilibre des recettes et des dépenses, et avait en définitive contracté des obligations que l'assistance de sa famille, de ses amis, les subsides que Humboldt avait obtenu du Roi étaient incapables d'éteindre. En outre, la discorde avait pénétré dans le groupe scientifique de Neuchâtel.

Ce fut un heureux appel en Amérique qui, dans ces circonstances, sauva une situation qui devenait de plus en plus critique. Aussi le savant professeur prèta-t-il l'oreille lorsqu'il lui fut adressé. A l'instigation du géologue anglais Lyell, un citoyen de Boston, M. John Lowell, le pria de venir donner des conférences dans cette ville. Afin de l'encourager dans sa résolution, Humboldt obtint pour lui de Frédéric-Guillaume IV une mission scientifique dans le Nouveau Monde et - le roi lui accorda une subvention annuelle de 8000 livres, soit environ fr, ir, ooo pendant deux ans, dans l'intention que les collections qu'il amasserait dans ce voyage seraient essentiellement destinées au Musée de Neuchâtel et les doubles seulement à celui de Berlin.

Au reste, en quittant Neuchâtel, Agassiz ne faisait que mettre à exécution un rêve de sa jeunesse, car il avait toujours désiré prendre part à un voyage de découvertes où il aurait l'occasion d'utiliser la surabondance de force et d'énergie qui débordait en lui. Et si l'Amérique l'attirait, c'est sans doute parce que ce pays convenait mieux que tout autre à son génie entreprenant et que son infatigable énergie devait y trouver son véritable champ d'action. Après bien des hésitations il accepta, mais sans avoir un instant l'idée qu'il ne reviendrait pas. Sa route était tracée, il savait ce qu'il allait faire de l'autre còté de l'Océan. Son but atteint, il retournerait en Europe où les perspectives d'avenir ne lui manqueAGASSIZ. -3 
raient pas. Il aimait trop sa petite patrie pour songer à lui dire un éternel adieu, et s'il partait joyeux à la pensée des choses nouvelles qu'il allait voir, c'était parce qu'il complait bien revenir.

Cela se passait au printemps de i 845 . Grand fut l'émoi à Neuchâtel lorsque le bruit se répandit que le professeur aimé allait nous quitter et que nous étions sur le point de perdre l'homme éminent qui nous avait fait une réputation à l'étranger el avait attiré sur nous l'attention du monde savant. Plusieurs avaient même la conviction que l'Amérique le retiendrait et qu'on ne le reverrait jamais. Le plus affecté était Louis Coulon, qui voyait avec inquiétude s'éloigner cet ami dont les promesses de retour ne parvenaient pas à le rassurer. C'est qu'il avait et à juste titre comme le pressentiment qu'une lumière allait s'éteindre chez nous pour briller sous un ciel qui lui fut plus favorable.

Agassiz passa encore l'hiver de r845 à i 846 à Neuchâtel, occupé à terminer différentes publications, retenu aussi par les démarches relatives à son remplacement à l'Académie. Il nous quitta les premiers jours de mars i 846 pour aller passer quelques mois à Paris et s'embarqua pour Boston en septembre, loin de se douter qu'en son absence une révolution emporterait sa chaire et qu'il allait trouver un établissement définitif par delà l’Océan.

Je renonce à suivre Agassiz en Amérique et à décrire la série de travaux qu'il entreprit aux États-Unis - travaux d'ordre plus spécialement zoologique, - où sa carrière a été sinon plus brillante, du moins aussi brillante qu'en Europe. On sait quelle fut sa destinée dans le Nouveau Monde, où il débarquait précédé par le prestige d'une réputation européenne, qui constituait à l'avance pour lui une condition de succès, et comment il y trouva un théâtre approprié à son génie entreprenant, ainsi que des ressources que l'Europe ne 
lui eùt jamais offertes. Et si dans le temps où il n'élait encore qu'un pauvre étudiant il a rèvé un Eldorado approprié à sa passion pour l'histoire naturelle, son rêve s'est trouvé pleinement réalisé à la fin de sa vie.

En I 848 - et cela avec autant de discernement que Louis Coulon en avait montré seize ans auparavant, - un généreux Américain, pénétré de l'utilité qu’il y aurait pour son pays à y retenir un homme de cette valeur, lui offrit de créer une chaire de zoologie et géologie à l'école scientifique du Harward College, à Cambridge. C'était l'année des révolutions. L'Académie venait d'être supprimée. Du côté de l'Europe tout semblait lui manquer, tandis que l'Amérique lui montrait l'aurore d'un avenir certain et lui permettait de s'affranchir des inquiétudes qui le poursuivaient. Aussi, séduit et touché par les marques significatives de sympathie qui lui sont témoignées, il abandonne la pensée d'un retour sur le vieux continent et met son activité, sa science, ses talents, au service de cette nation généreuse qui veut le garder. C'est ainsi qu'il quitta le modeste théâtre où il avait d'abord brillé et commença dans le Nouveau Monde une carrière dans laquelle il devait trouver des ressources encore plus grandes que celles qu'il poursuivait èn rêve. Et lorsque quelques amnées plus tard l'Université de Zurich et le Gouvernement français cherchèrent à le faire revenir en Europe, il répond qu'il est décidé à rester en Amérique, où il a la conviction de pouvoir exercer une influence plus réelle et plus étendue sur les progrès de la science, et cela non seulement par son enseignement de chaque jour, mais aussi par la réalisation de ce fameux Musée de zoologie comparée dont il avait muri le plan pendant tant d'années et au développement duquel il consacra toute son activité dernière.

Le champ de travail qu'Agassiz trouva aux États-Unis, les créations provoquées par sa présence, les sacrifices faits pour les soutenir, tous ces encouragements l'attachèrent à ce sol 
qu'il se proposait seulement de visiter. Il rencontra dans ce pays jeune, grand, généreux, des appuis providentiels qui lui permirent de réaliser des entreprises qu’il aurait considérées autrefois comme les rêves d'une imagination exaltée. Et un pareil rẻsultat n'était pas dû au hasard, mais bien à la noble et légitime influence exercée par l'intelligence supérieure du savant et les qualités aimables de l'homme. Encouragé par l'appui d'un pays qui met tout à sa disposition, il vit peu à peu se développer une brillante carrière de propagande scientifique, de voyages splendides, qui ont fixé sur lui l'attention. L'école de Cambridgye, le Musée Agassiz, l'école d'histoire naturelle Anderson, dans l'île de Penikese, comme aussi l'intérêt éveillé unixersellement pour la science dans le Nouveau Monde, sont les monuments durables de l'influence qu'il a exercée.

L'homme qui inspira une confiance si extraordinaire et provoqua de tels élans de générosité et de reconnaissance devait être nécessairement un homme de génie et de grand cœur. C'est dans une modeste habitation près de l'Université de Harward qu'il est mort le 18 décembre 1873 , d'une prostration rapide du système nerveux, épuisé par un labeur de tous les instants et au-dessus des forces de l'homme le plus vigou. reusement constitué. Sur sa tombe repose un bloc erratique, arraché à une des moraines du glacier de l'Aar, entouré de quelques sapins du. Jura, souvenirs humbles et touchants, donnés par son pays d'adoption au pays qui l'avait vu naître. Tandis que ce granit finira par se désagréger, l'œuvre d'Agassiz subsistera, elle vieillira sans doute, elle sera dépassée par des cuvres plus parfaites ou plus complètes, mais elle demeurera encore aux yeux de tous un monument du génie de l'homme. On se souvient que le grand naturaliste a laissé après lui un fils qui s'est montré digne de le comprendre et de lui succéder. Plein de vénération pour la mémoire de son père, il tient à conserver comme un précieux patrimoine ces tradi- 
tions d'amour et de respect pour le travail qui lui ont été si noblement léguées.

Parmi les savants dont la Suisse romande peut avec raison s'honorer, Agassiz est certainement un de ceux dont la réputation est le plus populaire. Mais il y a mieux. Ses prodigieuses capacités, son talent exceptionnel d'observation, la facilité avec laquelle il se mettait au fait de toutes les questions et abordait les sujets les plus divers, le grand mouvement intellectuel qu'il a développé partout oì il a vécu, la valeur de ses propres recherches, ont fait de son nom l'un des plus grands de la science au XIXe siècle. Avec cette belle assurance qui fut un des traits de sa nature expansive, il écrivail de Munich à son père, le I4 février I 829: "Je voudrais que l'on pût dire de Louis Agassiz, il fut le premier naturaliste de son siècle, bon citoyen et bon fils, aimé de tous ceux qui le connurent. Je sens en moi la force d'une génération entière pour travailler à ce but et je veux l'atteindre si les moyens ne me manquent pas... Il ne s'agit que de me faire un nom européen ; je suis dans le meilleur chemin pour y parvenir. ') Tels étaient, à vingt-deux ans, son programme et son rêve. Personne ne niera qu'ils se soient réalisés, el si Agassiz n'a pas été le premier, il a certainement été l'un des premiers naturalistes du siècle passé. N'était-ce pas assez pour justifier toutes ses ambitions?

C'est un beau et fortifiant spectacle que celui de cette activité intense, soutenue sans défaillance pendant de longues années. Un immense savoir, des découvertes nombreuses, des vues neuves et hardies, inspirées par'la pénétration de son esprit et mûries par la raison, une parole persuasive qui charmait ou captivait les âmes et les entrainait vers de hautes pensées, ont procuré à Agassiz l'estime et la réputation parmi ses contemporains, et une grande et heureuse influence dans le mouvement scientifique moderne. On vit chez lui la passion 
de l'étude aussi ardente dans les années de sa vieillesse qu'au début de sa carrière, une ambition extrème concentrée dans le désir de pénétrer les plus merveilleux phénomènes de la nature. Il ne s'est pas contenté d'étudier les animaux aujourd'hui vivants; il a écrit sur les animaux fossiles des ouvrages considérables, qui sont de véritables monuments scientifiques. Dela comparaison entre la faune vivante et les faunes éteintes, il a su tirer des conclusions aussi neuves que profondes relativement à la succession des formes organiques sur la terre. Son activité intellectuelle a tout embrassé ; il s'est associé a vec ardeur au mouvement de la zoologie moderne, qui poursuit la solution de problèmes nouveaux dans l'étude si longtemps négligée des animaux inférieurs et de l'embryologie comparée.

Comment un homme de ce mérite, un savant que des écoles plus grandes et plus importantes que celle de Neuchâtel auraient désiré posséder, a-t-il pu consentir à se fixer parmi nous ? C'est qu'il ne recherchait pas la fortune, car pendant. son séjour en Europe des offres brillantes lui étaient venues de Heidelberg, de Lausanne et de Genève, et malgré cela il resta fidèle à Neuchâtel où il avait trouvé un accueil chaleureux et sympathique. Nous n'oublions pas, en effet, de dire qu'il y rencontra un terrain bien préparé, des esprits curieux de s'instruire, de bonnes volontés prêtes à le seconder, des facilités particulières pour les travaux qu'il méditait et des collègues dévonés comme lui à la science. Au reste, un des traits particuliers qui frappent chez Agassiz, c'est son grand désintéressement dont il fit preuve durant toute sa vie, s'oubliant lui-même et consacrant toujours la totalité de ses ressources à atteindre le but élevé qu'il poursuivait; il était avant tout dévoué à la science, il s'y donna tout entier, sans réserve, et il eut toujours l'habileté de faire partager aux autres l'ardeur qui l'animait. Mettant à contribution les talents des uns, la bourse des autres, ajoutant à tout cela ses ressources, son temps et son génie, il est arrivé au résultat que 
chacun connaît et peut constater aujourd'hui. L'argent n'avait de valeur à ses yeux que parce qu'il sert à l'avancement de la science. Il l'a prouvé en ne laissant à sa mort aucune fortune, bien qu'il ait été en situation de gagner des sommes considérables s'il l'avait voulu.

A l'inverse de tant de savants, qui renferment obstinément leurs pensées dans les bornes étroites du monde sensible et ne voient dans la nature qu'une série d'évolutions de la matière, il fut pendant toute sa carrière un spiritualiste convaincu. C'est qu'après avoir sondé les secrets de la nature, après avoir voué à celte recherche le travail le plus ardu et le plus persévérant, et voulu le poursuivre avec les seules lumières de la science, il a trouvé à l'origine de tout un Dieu créateur. Le dernier cours qu'il donna à Neuchâtel était précisément destiné à exposer le plan de la Création et le dernier travail sorti de sa plume, et qui est devenu comme son testament scientifique, n'avait pas d'autre but que de réfuter les doctrines contre lesquelles protestait toute sa conception de la nature.

Agassiz a été en butte de son vivant et surtout après sa mort aux attaques les plus violentes de la part des champions de l'école transformiste qui, au lieu de se renfermer dans l'étroite et soigneuse recherche des faits, se livraient à la spéculation. Sans doute, plusieurs des idées qu'il a émises ont été abandonnées, mais les discussions auxquelles elles ont donné lieu ont été une source de progrès féconds et le temps est venu où la puissance et la profondeur de son intelligence ont été dignement appréciées, car l'œuvre qu’il a laissée est considérable.

Cette noble et sympathique figure serait-elle destinée à disparaitre dans le gouffre sans fond du passé? A Dieu ne plaise. Cette vie si bien remplie ne manque pas d'utiles enseignements, ne serait-ce que celui qui se dégage tout naturelle- 
ment d'une carrière où tout est dù à l'initiative d'une volonté énergique, où l'homme a conquis ses étapes les unes après les autres. Le temps qui efface tant de personnalités perpétue et entoure sans cesse d'un nouvel éclat le nom de ces hommes rares, qui semblent avoir révélé de nouveaux ressorts de l'intelligence et donné de nouvelles forces à la pensée. Et comme leur esprit, devançant leur siècle, avait surtout en vue la postérité, ce n’est aussi que de cette postérité qu’ils peuvent attendre tout ce qui leur est dù de reconnaissance et d'admiration.

La cérémonie d'aujourd'hui nous rappelle une période dejà lointaine, mais particulièrement brillante de notre histoire. Elle est pour nous une occasion de réveiller le souvenir d'une époque de travail et de ferveur dans tous les genres d'investigations scientifiques.

Qu'il est bon et salutaire de rappeler à la génération présente ce passé glorieux, non pour nous humilier et nous décourager, mais pour y puiser une saine émulation et chercher à ne pas démériter de nos anciens.

En ce jour anniversaire, inclinons-nous devant cette noble physionomie dont je viens de rappeler brièvement l'existence et rappelons-nous celui qui a été un serviteur éminent de la science, celui qui honora la Suisse et illustra Neuchâtel. 
